\title{
Existence Results for Fractional Integro-Differential Equations with Fractional Order Non-instantaneous Impulsive Conditions
}

\author{
A. Anguraj and S. Kanjanadevi \\ PSG College of Arts and Science, Coimbatore-641 014, Tamil Nadu, India \\ Email: angurajpsg@yahoo.com
}

\begin{abstract}
In this paper, we prove the existence results for fractional integro-differential equations with fractional order non-instantaneous impulsive conditions. We prove the existence of mild solutions by using the resolvent operator and fixed point theorem for condensing map.
\end{abstract}

Keywords: Fractional differential equations, fractional non-instantaneous impulsive conditions, resolvent operator.

\section{Introduction}

The theory of fractional differential equations has played an important role in the field of science and engineering, since many physical systems can be represented more accurately through fractional derivative formulation. The non-integer order differential equations have the ability to describe the real behavior and memory effects of the systems and processes. We can use the fractional calculus for the mathematical modeling of systems and processes in many fields of applications such as physics, chemistry, aerodynamics, electrodynamics of complex medium, viscoelasticity, heat conduction, electricity mechanics, control theory, and so forth. For more details about the fractional differential equations and their applications we refer to $[7,8,16,18,22,27]$.

The study of impulsive differential equations has more attention in recent years due to their applications. Most of the research papers deal with the existence of solutions for equations with instantaneous impulsive conditions, see $[1,2,3,9,19,20,24]$. This type of impulsive problems are an appropriate model for describing the processes which at certain moments change their state rapidly. Recently, E. Hernández and D. O'regan [12] introduced a new class of impulsive differential equations. In the model presented in [12], the impulses start abruptly at certain point of time and their actions continue with a finite time interval. The non-instantaneous impulsive systems are more suitable to study the dynamics of evolution processes in pharmacotheraphy. The existence of solutions for non-instantaneous impulsive fractional/ integer order differential equations has also been studied $[4,6,10,26,29,30]$.

On the other hand, we came to know from the semigroup theory that many authors used the concept of mild solutions inappropriately, see $[5,15,23,25]$. To make the concept of mild solutions more appropriate, E. Hernández et. al [13] treated abstract differential equations with fractional derivatives in time t, based on the well developed theory of resolvent operators for integral equations [28]. In [17] N Kosmatov studied the initial value problems of fractional order with fractional impulsive conditions. From the result in [17] and the application of fractional derivative we came to know that the fractional order non-instantaneous impulsive systems were more powerful than those with the integer order impulsive conditions.

In this present work, we study the existence of fractional integro-differential equations with fractional order non-instantaneous impulsive conditions of the form

$$
\begin{aligned}
{ }^{c} D^{\alpha} u(t) & =A u(t)+f(t, u(t), \mathcal{B} u(t)), t \in\left(s_{i}, t_{i+1}\right], \quad i=0,1, \cdots, N \\
{ }^{c} D^{\beta} u(t) & =g_{i}(t, u(t)), \quad t \in\left(t_{i}, s_{i}\right], i=1, \cdots, N \\
u(0) & =u_{0} \in X
\end{aligned}
$$

where $0<\beta<\alpha<1, A: \mathcal{D}(A) \subset X \rightarrow X$ is the infinitesimal generator of a $C_{0}$-semigroup of bounded linear operator $(S(t))_{t \geq 0}$ defined on a Banach space $(X,\|\cdot\|), 0=t_{0}=s_{0}<t_{1}<s_{1}<\cdots<s_{N}<t_{N+1}=$ 
1 are pre-fixed numbers, the functions $f:[0,1] \times X \times X \rightarrow X, g_{i}:\left(t_{i}, s_{i}\right] \times X \rightarrow X$ are continuous, $\mathcal{B}: C([0,1] ; X) \rightarrow C([0,1] ; X)$ is given by $\mathcal{B} u(t)=\int_{0}^{t} B(t, s) u(s) d s$ and $\{B(t, s): 1 \geq t \geq s \geq 0\}$ is a set of bounded linear operator on $X$ such that $B(t, \cdot) u \in C([0, t] ; X)$ and $B(\cdot, s) u \in C([s, 1] ; X)$ for all $t, s \in[0,1]$ and each $u \in X$.

We use the resolvent operator for integral equations to represents the mild solutions of the systems, which is more appropriate, and we use the fixed point technique for condensing map to prove the existence of results for the system (1)-(3).

\section{Preliminaries}

Let $X$ and $Y$ be Banach spaces and $\mathcal{L}(X, Y)$ denote the space of all bounded linear operators with norm $\|\cdot\|_{\mathcal{L}(X, Y)}$. The domain of $A$ is endowed with the graph norm $\|\cdot\|_{\mathcal{D}(A)}=\|x\|+\|A x\|$. In addition, $B_{r}(x, X)$ represents the closed ball with center at $x$ and radius $r$ in $X$. The space $C([0,1] ; X)$ denotes the space of all continuous functions with norm $\|\cdot\|_{C([0,1] ; X)}=\sup _{t \in[0,1]}\|x(t)\|_{X}$.

We introduce the space $P C(X)$ which is formed by all the functions $u:[0,1] \rightarrow X$ such that $u($.$) is$ continuous at $t \neq t_{i}, u\left(t_{i}^{-}\right)=u\left(t_{i}\right)$ and $u\left(t_{i}^{+}\right)$exists for all $i=1,2, \cdots, N . P C(X)$ is a Banach space with respect to the norm $\|u\|_{P C(X)}=\sup _{t \in[0,1]}\|u(t)\|$. For a function $u \in P C(X)$ and $i \in\{0,1, \cdots, N\}$, we introduce the function $\tilde{u}_{i} \in C\left(\left[t_{i}, t_{i+1}\right] ; X\right)$ given by

$$
\tilde{u}_{i}(t)=\left\{\begin{array}{lc}
u(t), & \text { for } t \in\left(t_{i}, t_{i+1}\right], \\
u\left(t_{i}^{+}\right), & \text {for } t=t_{i}
\end{array}\right.
$$

In addition, for $E \subseteq P C(X)$ and $i \in\{0,1, \cdots, N\}$, we use the notation $\tilde{E}_{i}$ for the set $\tilde{E}_{i}=\left\{\tilde{u}_{i}: u \in E\right\}$. We note the following Ascoli-Arzela type criteria.

Lemma 21. [11] A set $E \subseteq P C(X)$ is relatively compact in $P C(X)$ if and only if each set $\tilde{E}_{i}$ is relatively compact in $C\left(\left[t_{i}, t_{i+1}\right] ; X\right)$.

From [16], we know that Caputo fractional derivative of order $\alpha>0$ of a function $u$ is defined as follows:

$$
D^{\alpha} u(t)=I^{n-\alpha} D^{n} u(t), n=\lceil\alpha\rceil,
$$

where $I^{\alpha} u(t)=\frac{1}{\Gamma(\alpha)} \int_{0}^{t}(t-s)^{\alpha-1} u(s) d s$ is Riemann-Liouville fractional integral. Also, in general the Caputo derivative is a left inverse of $I^{\alpha}$ but not a right inverse, i.e., we have $D_{t}^{\alpha} I_{t}^{\alpha} u=u$ and $I_{t}^{\alpha} D_{t}^{\alpha} u(t)=$ $u(t)-u(0)$ for $0<\alpha<1$.

Now, we establish the equivalent integral equation of the system (1)-(3) to explore the mild solution of the fractional non-instantaneous impulsive system (1)-(3).

Apply fractional integral on both sides of (1)-(3), we get for $t \in\left(0, t_{1}\right]$,

$$
u(t)=u_{0}+\frac{1}{\Gamma(\alpha)} \int_{0}^{t}(t-s)^{\alpha-1}(A u(s)+f(s, u(s), \mathcal{B} u(t))) d s,
$$

for $t \in\left(t_{1}, s_{1}\right]$

$$
\begin{aligned}
u(t)= & u_{0}+\frac{1}{\Gamma(\beta)} \int_{t_{1}}^{t}(t-s)^{\beta-1} g_{i}(t, u(t)) d s \\
& +\frac{1}{\Gamma(\alpha)} \int_{0}^{t_{1}}\left(t_{1}-s\right)^{\alpha-1}(A u(s)+f(s, u(s), \mathcal{B} u(t))) d s
\end{aligned}
$$

for $t \in\left(s_{1}, t_{2}\right]$,

$$
\begin{aligned}
u(t)= & u_{0}+\frac{1}{\Gamma(\beta)} \int_{t_{1}}^{s_{1}}\left(s_{1}-s\right)^{\beta-1} g_{i}(t, u(t)) d s \\
& +\frac{1}{\Gamma(\alpha)} \int_{0}^{t_{1}}\left(t_{1}-s\right)^{\alpha-1}(A u(s)+f(s, u(s), \mathcal{B} u(t))) d s \\
& +\frac{1}{\Gamma(\alpha)} \int_{s_{1}}^{t}(t-s)^{\alpha-1}(A u(s)+f(s, u(s), \mathcal{B} u(t))) d s
\end{aligned}
$$


Repeating this procedure up to $i=N$, we get

$$
\begin{aligned}
u(t)= & u_{0}+\frac{1}{\Gamma(\alpha)} \sum_{i=0}^{N} \int_{s_{i}}^{t_{i+1}}\left(t_{i+1}-s\right)^{\alpha-1}(A u(s)+f(s, u(s), \mathcal{B} u(t))) d s \\
& +\frac{1}{\Gamma(\beta)} \sum_{i=0}^{N} \int_{t_{i}}^{s_{i}}\left(s_{i}-s\right)^{\beta-1} g_{i}(s, u(s)) d s \\
& +\frac{1}{\Gamma(\beta)} \int_{t_{i}}^{t}(t-s)^{\beta-1} g_{i}(s, u(s)) d s, t \in\left(t_{i}, s_{i}\right], i=1, \cdots, N
\end{aligned}
$$

and

$$
\begin{aligned}
u(t)= & u_{0}+\frac{1}{\Gamma(\beta)} \sum_{i=1}^{N} \int_{t_{i}}^{s_{i}}\left(s_{i}-s\right)^{\beta-1} g_{i}(t, u(t)) d s \\
& +\frac{1}{\Gamma(\alpha)} \sum_{i=0}^{N} \int_{s_{i}}^{t_{i+1}}\left(t_{i+1}-s\right)^{\alpha-1}(A u(s)+f(s, u(s), \mathcal{B} u(t))) d s \\
& +\frac{1}{\Gamma(\alpha)} \int_{s_{i}}^{t}(t-s)^{\alpha-1}(A u(s)+f(s, u(s), \mathcal{B} u(t))) d s, t \in\left(s_{i}, t_{i+1}\right], i=1, \cdots, N
\end{aligned}
$$

Now, we consider that the following Volterra integral equation,

$$
u(t)=\frac{1}{\Gamma(\alpha)} \int_{0}^{t}(t-s)^{\alpha-1} A u(s)+f(t), t \in[0, a]
$$

has an associated resolvent operator $(S(t))_{t \geq 0}$ on $X$ and $f \in C([0, a] ; X)$.

Definition 22. [28, Definition 1.3] A family $(S(t))_{t \geq 0} \subset \mathcal{B}(X)$ of bounded linear operators in $X$ is called resolvent for $(7)$ (or solution operator for $(7)$ ), if the following conditions are satisfied

(S1) $S(t)$ is strongly continuous on $\mathbb{R}^{+}$and $S(0)=I$,

(S2) $S(t)$ commutes with $A$, which means that $S(t) \mathcal{D}(A) \subset \mathcal{D}(A)$ and

$A S(t) x=S(t) A x$ for all $x \in \mathcal{D}(A)$ and $t \geq 0$

(S3) The resolvent equation holds

$$
S(t) x=x+\int_{0}^{t} \frac{(t-s)^{\alpha-1}}{\Gamma(\alpha)} A S(s) x d s, \text { for all } x \in \mathcal{D}(A), t \geq 0 .
$$

In this paper, we always assume that $(S(t))_{t \geq 0}$ is analytic [28, Chap. 2] and there is $\phi_{A} \in L_{l o c}^{1}\left(\mathbb{R}^{+}\right)$ such that $\left\|S^{\prime}(t) x\right\| \leq \phi_{A}(t)\|x\|_{\mathcal{D}(A)}$ a.e. on $\mathbb{R}^{+}$for each $x \in \mathcal{D}(A)$.

Definition 23. [28, Definition 1.1] A function $u \in C([0, a] ; X)$ is called a mild solution of $(7)$ on $[0, a]$ if $\frac{1}{\Gamma(\alpha)} \int_{0}^{t}(t-s)^{\alpha-1} u(s) d s \in C([0, a] ; \mathcal{D}(A))$ and

$$
u(t)=\frac{A}{\Gamma(\alpha)} \int_{0}^{t}(t-s)^{\alpha-1} u(s) d s+f(t) \text { on }[0, a] .
$$

Lemma 24. [28, Section 1.2] Suppose (7) admits a resolvent $S(t)$ and let $f \in C([0, a] ; X)$. Then

(i) if $u \in C([0, a] ; X)$ is a mild solution of (7), then $\int_{0}^{t} S(t-s) f(s) d s$ is continuously differentiable on $[0, a]$ and

$$
u(t)=\frac{d}{d t} \int_{0}^{t} S(t-s) f(s) d s, t \in[0, a]
$$

in particular, mild solution of (7) are unique, 
(ii) if $f \in C([0, a] ; \mathcal{D}(A))$ and $S(t)$ is differentiable resolvent then

$$
u(t)=f(t)+\int_{0}^{t} S^{\prime}(t-s) f(s) d s, t \in[0, a],
$$

is a mild solution of ( 7$)$.

In the following section, we prove the existence of results for (1)-(3) by using fixed point theorem via condensing map.

\section{$3 \quad$ Existence Results}

In this section, we prove the existence of mild solution of the system (1)-(3). We have already shown that the fractional differential equations (1)-(3) are equivalent to the fractional integral equations (4)-(6).

First, we introduce the concept of mild solution for (1)-(3) using the preliminary results on integral equation, Definition 23

Definition 31. A function $u \in P C([0,1] ; X)$ is said to be a mild solution of (1)-(3) on $[0,1]$,

(i) if $\frac{1}{\Gamma(\alpha)} \int_{0}^{t}(t-s)^{\alpha-1} u(s) d s \in \mathcal{D}(A)$ for all $t \in\left[0, t_{1}\right]$ and

$$
\begin{aligned}
u(t)= & u_{0}+\frac{A}{\Gamma(\alpha)} \int_{0}^{t}(t-s)^{\alpha-1} u(s) d s \\
& +\frac{1}{\Gamma(\alpha)} \int_{0}^{t}(t-s)^{\alpha-1} f(s, u(s), \mathcal{B} u(s)) d s, t \in\left[0, t_{1}\right]
\end{aligned}
$$

(ii) if $\frac{1}{\Gamma(\alpha)} \sum_{i=0}^{N} \int_{s_{i}}^{t_{i+1}}\left(t_{i+1}-s\right)^{\alpha-1} u(s) d s \in \mathcal{D}(A)$ for all $t \in\left(t_{i}, s_{i}\right]$, and

$$
\begin{aligned}
u(t)= & u_{0}+\frac{A}{\Gamma(\alpha)} \sum_{i=0}^{N} \int_{s_{i}}^{t_{i+1}}\left(t_{i+1}-s\right)^{\alpha-1} u(s) d s \\
& +\frac{1}{\Gamma(\alpha)} \sum_{i=0}^{N} \int_{s_{i}}^{t_{i+1}}\left(t_{i+1}-s\right)^{\alpha-1} f(s, u(s), \mathcal{B} u(s)) d s \\
& +\frac{1}{\Gamma(\beta)} \sum_{i=0}^{N} \int_{t_{i}}^{s_{i}}\left(s_{i}-s\right)^{\beta-1} g_{i}(s, u(s)) d s \\
& +\frac{1}{\Gamma(\beta)} \int_{t_{i}}^{t}(t-s)^{\beta-1} g_{i}(s, u(s)) d s, t \in\left(t_{i}, s_{i}\right], i=1, \cdots, N
\end{aligned}
$$

(iii) if $\frac{1}{\Gamma(\alpha)}\left(\sum_{i=0}^{N} \int_{s_{i}}^{t_{i+1}}\left(t_{i+1}-s\right)^{\alpha-1} u(s) d s+\int_{s_{i}}^{t}(t-s)^{\alpha-1} u(s) d s\right) \in \mathcal{D}(A)$ for all $t \in\left(s_{i}, t_{i+1}\right]$, and

$$
\begin{aligned}
u(t)= & u_{0}+\frac{1}{\Gamma(\beta)} \sum_{i=1}^{N} \int_{t_{i}}^{s_{i}}\left(s_{i}-s\right)^{\beta-1} g_{i}(s, u(s)) d s \\
& +\frac{A}{\Gamma(\alpha)}\left(\sum_{i=0}^{N} \int_{s_{i}}^{t_{i+1}}\left(t_{i+1}-s\right)^{\alpha-1} u(s) d s+\int_{s_{i}}^{t}(t-s)^{\alpha-1} u(s) d s\right) \\
& +\frac{1}{\Gamma(\alpha)} \sum_{i=0}^{N} \int_{s_{i}}^{t_{i+1}}\left(t_{i+1}-s\right)^{\alpha-1} f(s, u(s), \mathcal{B} u(s)) d s \\
& +\frac{1}{\Gamma(\alpha)} \int_{s_{i}}^{t}(t-s)^{\alpha-1} f(s, u(s), \mathcal{B} u(s)) d s, t \in\left(s_{i}, t_{i+1}\right], i=1, \cdots, N
\end{aligned}
$$


Suppose that (4)-(6) admits a differential resolvent operator $(S(t))_{t \geq 0}$ and if $f \in C([0,1] ; \mathcal{D}(A))$, then from Lemma 24, we have the mild solutions of (1)-(3) as

$$
\begin{aligned}
u(t)= & u_{0}+\frac{1}{\Gamma(\alpha)} \int_{0}^{t}(t-s)^{\alpha-1} f(s, u(s), \mathcal{B} u(s)) d s \\
& +\int_{0}^{t} S^{\prime}(t-s)\left(\frac{1}{\Gamma(\alpha)} \int_{0}^{s}(s-\tau)^{\alpha-1} f(\tau, u(\tau), \mathcal{B} u(\tau)) d \tau\right) d s, \text { for } t \in\left[0, t_{1}\right], \\
u(t)= & u_{0}+\frac{1}{\Gamma(\alpha)} \sum_{i=0}^{N} \int_{s_{i}}^{t_{i+1}}\left(t_{i+1}-s\right)^{\alpha-1} f(s, u(s), \mathcal{B} u(s)) d s \\
& +\frac{1}{\Gamma(\beta)} \sum_{i=0}^{N} \int_{t_{i}}^{s_{i}}\left(s_{i}-s\right)^{\beta-1} g_{i}(s, u(s)) d s+\frac{1}{\Gamma(\beta)} \int_{t_{i}}^{t}(t-s)^{\beta-1} g_{i}(s, u(s)) d s \\
& +\int_{t_{i}}^{t} S^{\prime}(t-s)\left(u_{0}+\frac{1}{\Gamma(\alpha)} \sum_{i=0}^{N} \int_{s_{i}}^{t_{i+1}}\left(t_{i+1}-\tau\right)^{\alpha-1} f(\tau, u(\tau), \mathcal{B} u(\tau)) d \tau\right. \\
& +\frac{1}{\Gamma(\beta)} \sum_{i=0}^{N} \int_{t_{i}}^{s_{i}}\left(s_{i}-\tau\right)^{\beta-1} g_{i}(\tau, u(\tau)) d \tau \\
& \left.+\frac{1}{\Gamma(\beta)} \int_{t_{i}}^{s}(s-\tau)^{\beta-1} g_{i}(\tau, u(\tau)) d \tau\right) d s, \text { for } t \in\left(t_{i}, s_{i}\right],
\end{aligned}
$$

and

$$
\begin{aligned}
u(t)= & u_{0}+\frac{1}{\Gamma(\beta)} \sum_{i=1}^{N} \int_{t_{i}}^{s_{i}}\left(s_{i}-s\right)^{\beta-1} g_{i}(s, u(s)) d s \\
& +\frac{1}{\Gamma(\alpha)} \sum_{i=0}^{N} \int_{s_{i}}^{t_{i+1}}\left(t_{i+1}-s\right)^{\alpha-1} f(s, u(s), \mathcal{B} u(s)) d s \\
& +\frac{1}{\Gamma(\alpha)} \int_{s_{i}}^{t}(t-s)^{\alpha-1} f(s, u(s), \mathcal{B} u(s)) d s \\
& +\int_{s_{i}}^{t} S^{\prime}(t-s)\left(u_{0}+\frac{1}{\Gamma(\beta)} \sum_{i=1}^{N} \int_{t_{i}}^{s_{i}}\left(s_{i}-\tau\right)^{\beta-1} g_{i}(\tau, u(\tau)) d \tau\right. \\
& +\frac{1}{\Gamma(\alpha)} \sum_{i=0}^{N} \int_{s_{i}}^{t_{i+1}}\left(t_{i+1}-\tau\right)^{\alpha-1} f(\tau, u(\tau), \mathcal{B} u(\tau)) d \tau \\
& \left.+\frac{1}{\Gamma(\alpha)} \int_{s_{i}}^{s}(s-\tau)^{\alpha-1} f(\tau, u(\tau), \mathcal{B} u(\tau)) d \tau\right) d s, \text { for } t \in\left(s_{i}, t_{i+1}\right]
\end{aligned}
$$

for each $i=1, \cdots, N$.

For the existence of our main result, we assume the following hypotheses:

(H1) The function $f:[0,1] \times X \times X \rightarrow \mathcal{D}(A)$ is continuous and there exists a function $m_{f} \in C\left([0,1] ; \mathbb{R}^{+}\right)$ and a non-decreasing function $W:[0, \infty) \rightarrow(0, \infty)$ such that $\|f(t, u, v)\|_{\mathcal{D}(A)} \leq m_{f}(t) W(\|u\|+\|v\|)$, for all $t \in[0,1], u, v \in X$.

(H2) The function $g_{i}:\left(t_{i}, s_{i}\right] \times X \rightarrow \mathcal{D}(A)$ is continuous and there exists $L_{g_{i}} \in C\left(\left(t_{i}, s_{i}\right] ; \mathbb{R}^{+}\right)$such that $\left\|g_{i}(t, u)-g_{i}(t, v)\right\|_{\mathcal{D}(A)} \leq L_{g_{i}}(t)\|u-v\|$, for all $t \in\left(t_{i}, s_{i}\right], u, v \in X$.

Remark 32. In this work, $i_{c}$ denotes the inclusion map from $\mathcal{D}(A)$ into $X$ and we assume that $K=$ $\sup _{t \in[0,1]} \int_{0}^{t}\|B(t, s)\|_{\mathcal{L}(X)} d s$ is finite.

Lemma 33. [14] Assume $S(t)$ is compact for all $t>0$. Then $S^{\prime}(t)$ is compact for all $t>0$ and the inclusion map $i_{c}: \mathcal{D}(A) \rightarrow X$ is compact. 
Theorem 34. Assume that the hypotheses $(H 1)$ and $(H 2)$ are satisfied, $x_{0} \in \mathcal{D}(A)$ and $S(t)$ is compact for all $t>0$. If

$$
\begin{aligned}
& \left(\frac{t_{1}^{\alpha}}{\alpha \Gamma(\alpha)}\left\|m_{f}\right\| \limsup _{r \rightarrow \infty} \frac{1}{r} W((1+K) r)\right)\left(1+\left\|\phi_{A}\right\|_{L^{1}\left(\left[0, t_{1}\right] ; \mathbb{R}^{+}\right)}\right) \leq 1 \\
& \left(1+\left\|\phi_{A}\right\|_{L^{1}\left(\left(t_{i}, s_{i}\right] ; \mathbb{R}^{+}\right)}\right)\left(\frac{\sum_{i=0}^{N}\left(t_{i+1}-s_{i}\right)^{\alpha}}{\alpha \Gamma(\alpha)}\left\|m_{f}\right\| \limsup _{r \rightarrow \infty} \frac{1}{r} W((1+K) r)\right. \\
& \left.\quad+\left(\frac{\sum_{i=0}^{N}\left(s_{i}-t_{i}\right)^{\beta}}{\beta \Gamma(\beta)}+\frac{\left(s_{i}\right)^{\beta}}{\beta \Gamma(\beta)}\right) L_{g_{i}} r\right) \leq 1 \\
& \left(1+\left\|\phi_{A}\right\|_{L^{1}\left(\left(s_{i}, t_{i+1}\right] ; \mathbb{R}^{+}\right)}\right)\left(\frac{\sum_{i=0}^{N}\left(s_{i}-t_{i}\right)^{\beta}}{\beta \Gamma(\beta)} L_{g_{i}} r\right. \\
& \left.\quad+\left(\sum_{i=0}^{N} \frac{\left(t_{i+1}-s_{i}\right)^{\alpha}}{\alpha \Gamma(\alpha)}+\frac{\left.t_{i+1}^{\alpha}\right)}{\alpha \Gamma(\alpha)}\right)\left\|m_{f}\right\| \limsup _{r \rightarrow \infty} \frac{1}{r} W((1+K) r)\right) \leq 1
\end{aligned}
$$

then there exists at least one mild solution of the problem (1)-(3).

Proof. Choose $r>0$,

$$
\begin{aligned}
& \left(\left\|u_{0}\right\|+\frac{t_{1}^{\alpha}}{\alpha \Gamma(\alpha)}\left\|m_{f}\right\| W((1+K) s)\right)\left(1+\left\|\phi_{A}\right\|_{L^{1}\left(\left[0, t_{1}\right] ; \mathbb{R}^{+}\right)}\right) \leq s \\
& \left(1+\left\|\phi_{A}\right\|_{L^{1}\left(\left(t_{i}, s_{i}\right] ; \mathbb{R}^{+}\right)}\right)\left(\left\|u_{0}\right\|+\frac{\sum_{i=0}^{N}\left(t_{i+1}-s_{i}\right)^{\alpha}}{\alpha \Gamma(\alpha)}\left\|m_{f}\right\| W((1+K) s)\right. \\
& \left.+\left(\frac{\sum_{i=0}^{N}\left(s_{i}-t_{i}\right)^{\beta}}{\beta \Gamma(\beta)}+\frac{\left(s_{i}\right)^{\beta}}{\beta \Gamma(\beta)}\right)\left(L_{g_{i}} s+\left\|g_{i}(\cdot, 0)\right\|\right)\right) \leq s \\
& \left(1+\left\|\phi_{A}\right\|_{L^{1}\left(\left(s_{i}, t_{i+1}\right] ; \mathbb{R}^{+}\right)}\right)\left(\left\|u_{0}\right\|+\frac{\sum_{i=0}^{N}\left(s_{i}-t_{i}\right)^{\beta}}{\beta \Gamma(\beta)}\left(L_{g_{i}} s+\left\|g_{i}(\cdot, 0)\right\|\right)\right. \\
& \left.+\left(\sum_{i=0}^{N} \frac{\left(t_{i+1}-s_{i}\right)^{\alpha}}{\alpha \Gamma(\alpha)}+\frac{t_{i+1}^{\alpha}}{\alpha \Gamma(\alpha)}\right)\left\|m_{f}\right\| W((1+K) s)\right) \leq s
\end{aligned}
$$

for all $s \geq r$.

Transform the problem (1)-(3) into fixed point problem and consider the operator $\Gamma: B_{r}(0, P C([0,1] ; X)) \rightarrow$ $P C([0,1] ; X)$ defined by

$$
\Gamma u(t)=\left\{\begin{array}{l}
u_{0}+\frac{1}{\Gamma(\alpha)} \int_{0}^{t}(t-s)^{\alpha-1} f(s, u(s), \mathcal{B} u(s)) d s \\
+\int_{0}^{t} S^{\prime}(t-s)\left(u_{0}+\frac{1}{\Gamma(\alpha)} \int_{0}^{s}(s-\tau)^{\alpha-1} f(\tau, u(\tau), \mathcal{B} u(\tau)) d \tau\right) d s, t \in\left[0, t_{1}\right] \\
u_{0}+\frac{1}{\Gamma(\alpha)} \sum_{i=0}^{N} \int_{s_{i}}^{t_{i+1}}\left(t_{i+1}-s\right)^{\alpha-1} f(s, u(s), \mathcal{B} u(s)) d s \\
+\frac{1}{\Gamma(\beta)} \sum_{i=0}^{N} \int_{t_{i}}^{s_{i}}\left(s_{i}-s\right)^{\beta-1} g_{i}(s, u(s)) d s+\frac{1}{\Gamma(\beta)} \int_{t_{i}}^{t}(t-s)^{\beta-1} g_{i}(s, u(s)) d s \\
+\int_{t_{i}}^{t} S^{\prime}(t-s)\left(u_{0}+\frac{1}{\Gamma(\alpha)} \sum_{i=0}^{N} \int_{s_{i}}^{t_{i+1}}\left(t_{i+1}-\tau\right)^{\alpha-1} f(\tau, u(\tau), \mathcal{B} u(\tau)) d \tau\right. \\
+\frac{1}{\Gamma(\beta)} \sum_{i=0}^{N} \int_{t_{i}}^{s_{i}}\left(s_{i}-\tau\right)^{\beta-1} g_{i}(\tau, u(\tau)) d \tau \\
\left.+\frac{1}{\Gamma(\beta)} \int_{t_{i}}^{s}(s-\tau)^{\beta-1} g_{i}(\tau, u(\tau)) d \tau\right) d s, t \in\left(t_{i}, s_{i}\right] \\
u_{0}+\frac{1}{\Gamma(\beta)} \sum_{i=1}^{N} \int_{t_{i}}^{s_{i}}\left(s_{i}-s\right)^{\beta-1} g_{i}(s, u(s)) d s \\
+\frac{1}{\Gamma(\alpha)} \sum_{i=0}^{N} \int_{s_{i}}^{t_{i+1}}\left(t_{i+1}-s\right)^{\alpha-1} f(s, u(s), \mathcal{B} u(s)) d s \\
+\frac{1}{\Gamma(\alpha)} \int_{s_{i}}^{t}(t-s)^{\alpha-1} f(s, u(s), \mathcal{B} u(s)) d s \\
+\int_{s_{i}}^{t} S^{\prime}(t-s)\left(u_{0}+\frac{1}{\Gamma(\beta)} \sum_{i=1}^{N} \int_{t_{i}}^{s_{i}}\left(s_{i}-\tau\right)^{\beta-1} g_{i}(\tau, u(\tau)) d \tau\right. \\
+\frac{1}{\Gamma(\alpha)} \sum_{i=0}^{N} \int_{s_{i}}^{t_{i+1}}\left(t_{i+1}-\tau\right)^{\alpha-1} f(\tau, u(\tau), \mathcal{B} u(\tau)) d \tau \\
\left.+\frac{1}{\Gamma(\alpha)} \int_{s_{i}}^{s}(s-\tau)^{\alpha-1} f(\tau, u(\tau), \mathcal{B} u(\tau)) d \tau\right) d s, t \in\left(s_{i}, t_{i+1}\right]
\end{array}\right.
$$


for each $i=1, \cdots, N$.

From the assumption on $f$ and $g_{i}$, we see that

$$
\begin{aligned}
& \int_{0}^{t}\left\|S^{\prime}(t-s)\left(u_{0}+\frac{1}{\Gamma(\alpha)} \int_{0}^{s}(s-\tau)^{\alpha-1} f(\tau, u(\tau), \mathcal{B} u(\tau)) d \tau\right) d s\right\| \\
& \leq \int_{0}^{t} \phi_{A}(t-s)\left\|u_{0}\right\|_{\mathcal{D}(A)} d s+\frac{1}{\Gamma(\alpha)} \int_{0}^{t} \phi_{A}(t-s) \int_{0}^{s}(s-\tau)^{\alpha-1}\|f(\tau, u(\tau), \mathcal{B} u(\tau))\|_{\mathcal{D}(A)} d \tau d s \\
& \leq\left(\left\|u_{0}\right\|_{\mathcal{D}(A)}+\frac{t_{1}^{\alpha}}{\alpha \Gamma(\alpha)}\|f(\tau, u(\tau), \mathcal{B} u(\tau))\|_{\mathcal{D}(A)}\right)\left\|\phi_{A}\right\|_{L^{1}\left(\left[0, t_{1}\right] ; \mathbb{R}^{+}\right)}
\end{aligned}
$$

which implies that $s \rightarrow \int_{0}^{t} S^{\prime}(t-s)\left(u_{0}+\frac{1}{\Gamma(\alpha)} \int_{0}^{s}(s-\tau)^{\alpha-1} f(\tau, u(\tau), \mathcal{B} u(\tau)) d \tau\right) d s$ is integrable on $\left[0, t_{1}\right]$.

Consider for the interval $\left(s_{i}, t_{i+1}\right], i=1, \cdots, N$,

$$
\begin{aligned}
& \int_{s_{i}}^{t} \| S^{\prime}(t-s)\left(u_{0}+\frac{1}{\Gamma(\beta)} \sum_{i=1}^{N} \int_{t_{i}}^{s_{i}}\left(s_{i}-\tau\right)^{\beta-1} g_{i}(\tau, u(\tau)) d \tau\right. \\
& +\frac{1}{\Gamma(\alpha)} \sum_{i=0}^{N} \int_{s_{i}}^{t_{i+1}}\left(t_{i+1}-\tau\right)^{\alpha-1} f(\tau, u(\tau), \mathcal{B} u(\tau)) d \tau \\
& \left.+\frac{1}{\Gamma(\alpha)} \int_{s_{i}}^{s}(s-\tau)^{\alpha-1} f(\tau, u(\tau), \mathcal{B} u(\tau)) d \tau\right) \| d s \\
& \leq\left\|u_{0}\right\|_{\mathcal{D}}\left\|\phi_{A}\right\|+\frac{1}{\Gamma(\beta)} \int_{s_{i}}^{t} \phi_{A}(t-s) \sum_{i=1}^{N} \int_{t_{i}}^{s_{i}}\left(s_{i}-\tau\right)^{\beta-1}\left\|g_{i}(\tau, u(\tau))\right\|_{\mathcal{D}} d \tau d s \\
& +\frac{1}{\Gamma(\alpha)} \int_{s_{i}}^{t} \phi_{A}(t-s) \sum_{i=0}^{N} \int_{s_{i}}^{t_{i+1}}\left(t_{i+1}-\tau\right)^{\alpha-1}\|f(\tau, u(\tau), \mathcal{B} u(\tau))\|_{\mathcal{D}} d \tau d s \\
& +\frac{1}{\Gamma(\alpha)} \int_{s_{i}}^{t} \phi_{A}(t-s) \int_{s_{i}}^{s}(s-\tau)^{\alpha-1}\|f(\tau, u(\tau), \mathcal{B} u(\tau))\|_{\mathcal{D}} d \tau d s \\
& \leq\left(\left\|u_{0}\right\|_{\mathcal{D}}+\frac{1}{\beta \Gamma(\beta)} \sum_{i=1}^{N}\left(s_{i}-t_{i}\right)^{\beta}\left\|g_{i}(\tau, u(\tau))\right\|_{\mathcal{D}}\right. \\
& \left.+\frac{1}{\alpha \Gamma(\alpha)}\left(\sum_{i=0}^{N}\left(t_{i+1}-s_{i}\right)^{\alpha}+t_{i+1}^{\alpha}\right)\|f(\tau, u(\tau), \mathcal{B} u(\tau))\|_{\mathcal{D}}\right)\left\|\phi_{A}\right\|_{L^{1}\left(\left[s_{i}, t_{i+1}\right] ; \mathbb{R}^{+}\right)},
\end{aligned}
$$

then the function $s \rightarrow \int_{s_{i}}^{t} S^{\prime}(t-s)\left(u_{0}+\frac{1}{\Gamma(\beta)} \sum_{i=1}^{N} \int_{t_{i}}^{s_{i}}\left(s_{i}-\tau\right)^{\beta-1} g_{i}(\tau, u(\tau)) d \tau\right.$ $\left.+\frac{1}{\Gamma(\alpha)} \sum_{i=0}^{N} \int_{s_{i}}^{t_{i+1}}\left(t_{i+1}-\tau\right)^{\alpha-1} f(\tau, u(\tau), \mathcal{B} u(\tau)) d \tau+\frac{1}{\Gamma(\alpha)} \int_{s_{i}}^{s}(s-\tau)^{\alpha-1} f(\tau, u(\tau), \mathcal{B} u(\tau)) d \tau\right) d s$ is integrable.

Finally, consider for the interval $\left(t_{i}, s_{i}\right], i=1, \cdots, N$

$$
\begin{aligned}
& \int_{t_{i}}^{t} \| S^{\prime}(t-s)\left(u_{0}+\frac{1}{\Gamma(\alpha)} \sum_{i=0}^{N} \int_{s_{i}}^{t_{i+1}}\left(t_{i+1}-\tau\right)^{\alpha-1} f(\tau, u(\tau), \mathcal{B} u(\tau)) d \tau\right. \\
& \left.+\frac{1}{\Gamma(\beta)} \sum_{i=0}^{N} \int_{t_{i}}^{s_{i}}\left(s_{i}-\tau\right)^{\beta-1} g_{i}(\tau, u(\tau)) d \tau+\frac{1}{\Gamma(\beta)} \int_{t_{i}}^{s}(s-\tau)^{\beta-1} g_{i}(\tau, u(\tau)) d \tau\right) \| d s \\
& \leq\left\|u_{0}\right\|_{\mathcal{D}}\left\|\phi_{A}\right\|+\frac{1}{\Gamma(\alpha)} \int_{t_{i}}^{t} \phi_{A}(t-s) \sum_{i=0}^{N} \int_{s_{i}}^{t_{i+1}}\left(t_{i+1}-\tau\right)^{\alpha-1}\|f(\tau, u(\tau), \mathcal{B} u(\tau))\|_{\mathcal{D}} d \tau d s \\
& +\frac{1}{\Gamma(\beta)} \int_{t_{i}}^{t} \phi_{A}(t-s) \sum_{i=0}^{N} \int_{t_{i}}^{s_{i}}\left(s_{i}-\tau\right)^{\beta-1}\left\|g_{i}(\tau, u(\tau))\right\|_{\mathcal{D}} d \tau d s \\
& +\frac{1}{\Gamma(\beta)} \int_{t_{i}}^{t} \phi_{A}(t-s) \int_{t_{i}}^{s}(s-\tau)^{\beta-1}\left\|g_{i}(\tau, u(\tau))\right\|_{\mathcal{D}} d \tau d s
\end{aligned}
$$




$$
\begin{aligned}
\leq & \left(\left\|u_{0}\right\|_{\mathcal{D}}+\frac{1}{\alpha \Gamma(\alpha)} \sum_{i=0}^{N}\left(t_{i+1}-s_{i}\right)^{\alpha}\|f(\tau, u(\tau), \mathcal{B} u(\tau))\|_{\mathcal{D}}\right. \\
& +\frac{1}{\beta \Gamma(\beta)}\left(\sum_{i=0}^{N}\left(s_{i}-t_{i}\right)^{\beta}+s_{i}^{\beta}\left\|g_{i}(\tau, u(\tau))\right\|_{\mathcal{D}}\right)\left\|\phi_{A}\right\|_{L^{1}\left(\left(t_{i}, s_{i}\right] ; \mathbb{R}^{+}\right)}
\end{aligned}
$$

From this result, we see that $s \rightarrow \int_{t_{i}}^{t} S^{\prime}(t-s)\left(u_{0}+\frac{1}{\Gamma(\alpha)} \sum_{i=0}^{N} \int_{s_{i}}^{t_{i+1}}\left(t_{i+1}-\tau\right)^{\alpha-1} f(\tau, u(\tau), \mathcal{B} u(\tau)) d \tau\right.$ $\left.+\frac{1}{\Gamma(\beta)} \sum_{i=0}^{N} \int_{t_{i}}^{s_{i}}\left(s_{i}-\tau\right)^{\beta-1} g_{i}(\tau, u(\tau)) d \tau+\frac{1}{\Gamma(\beta)} \int_{t_{i}}^{s}(s-\tau)^{\beta-1} g_{i}(\tau, u(\tau)) d \tau\right) d s$ is integrable on $\left[s_{i}, t\right]$ for all $t \in\left(t_{i}, s_{i}\right]$. This implies that $\Gamma$ is well defined.

Our aim to prove that $\Gamma$ is a condensing map from $B_{r}(0, P C(X))$ into $B_{r}(0, P C(X))$. First we show that $\Gamma$ has values in $B_{r}(0, P C(X))$.

Let $u \in B_{r}(0, P C(X))$. For $i \geq 1$ and $t \in\left(s_{i}, t_{i+1}\right]$, we get

$$
\begin{aligned}
& \|\Gamma u(t)\| \leq\left\|u_{0}\right\|+\frac{1}{\Gamma(\beta)} \sum_{i=0}^{N} \int_{t_{i}}^{s_{i}}\left(s_{i}-s\right)^{\beta-1}\left(\left\|g_{i}(s, u(s))-g_{i}(s, 0)\right\|+\left\|g_{i}(s, 0)\right\|\right) d s \\
& +\frac{1}{\Gamma(\alpha)} \sum_{i=0}^{N} \int_{s_{i}}^{t_{i+1}}\left(t_{i+1}-s\right)^{\alpha-1}\|f(s, u(s), \mathcal{B} u(s))\| d s \\
& +\frac{1}{\Gamma(\alpha)} \int_{s_{i}}^{t}(t-s)^{\alpha-1}\|f(s, u(s), \mathcal{B} u(s))\| d s+\int_{s_{i}}^{t}\|S(t-s)\|\left(\left\|u_{0}\right\|\right. \\
& \left.+\frac{1}{\Gamma(\beta)} \sum_{i=0}^{N} \int_{t_{i}}^{s_{i}}\left(s_{i}-\tau\right)^{\beta-1}\left(\left\|g_{i}(\tau, u(\tau))-g_{i}(\tau, 0)\right\|+\left\|g_{i}(\tau, 0)\right\|\right) d \tau\right) \\
& +\frac{1}{\Gamma(\alpha)} \sum_{i=0}^{N} \int_{s_{i}}^{t_{i+1}}\left(t_{i+1}-\tau\right)^{\alpha-1}\|f(\tau, u(\tau), \mathcal{B} u(\tau))\| d \tau \\
& \left.+\frac{1}{\Gamma(\alpha)} \int_{s_{i}}^{s}(s-\tau)^{\alpha-1}\|f(\tau, u(\tau), \mathcal{B} u(\tau))\| d \tau\right) d s \\
& \leq\left\|u_{0}\right\|+\frac{1}{\Gamma(\beta)} \sum_{i=0}^{N} \int_{t_{i}}^{s_{i}}\left(s_{i}-s\right)^{\beta-1}\left(\left\|L_{g_{i}}\right\|\|u\|+\left\|g_{i}(\cdot, 0)\right\|\right) d s \\
& +\frac{1}{\Gamma(\alpha)} \sum_{i=0}^{N} \int_{s_{i}}^{t_{i+1}}\left(t_{i+1}-s\right)^{\alpha-1} m_{f}(s) W(\|u\|+\|\mathcal{B} u\|) d s \\
& +\frac{1}{\Gamma(\alpha)} \int_{s_{i}}^{t}(t-s)^{\alpha-1} m_{f}(s) W(\|u\|+\|\mathcal{B} u\|) d s \\
& +\int_{s_{i}}^{t} \phi_{A}(t-s)\left(\left\|u_{0}\right\|+\frac{1}{\Gamma(\beta)} \sum_{i=0}^{N} \int_{t_{i}}^{s_{i}}\left(s_{i}-s\right)^{\beta-1}\left(\left\|L_{g_{i}}\right\|\|u\|+\left\|g_{i}(\cdot, 0)\right\|\right) d \tau\right. \\
& +\frac{1}{\Gamma(\alpha)} \sum_{i=0}^{N} \int_{s_{i}}^{t_{i+1}}\left(t_{i+1}-\tau\right)^{\alpha-1} m_{f}(\tau) W(\|u\|+\|\mathcal{B} u\|) d \tau \\
& \left.+\frac{1}{\Gamma(\alpha)} \int_{s_{i}}^{s}(s-\tau)^{\alpha-1} m_{f}(\tau) W(\|u\|+\|\mathcal{B} u\|) d \tau\right) \\
& \leq\left(1+\left\|\phi_{A}\right\|_{L^{1}\left(\left(s_{i}, t_{i+1}\right] ; \mathbb{R}^{+}\right)}\right)\left(\left\|u_{0}\right\|+\frac{\sum_{i=0}^{N}\left(s_{i}-t_{i}\right)^{\beta}}{\beta \Gamma(\beta)}\left(L_{g_{i}} r+\left\|g_{i}(\cdot, 0)\right\|\right)\right. \\
& \left.+\left(\sum_{i=0}^{N} \frac{\left(t_{i+1}-s_{i}\right)^{\alpha}}{\alpha \Gamma(\alpha)}+\frac{t_{i+1}^{\alpha}}{\alpha \Gamma(\alpha)}\right)\left\|m_{f}\right\| W((1+K) r)\right) \\
& \leq r
\end{aligned}
$$


which implies that $\|\Gamma u\|_{C\left(\left(s_{i}, t_{i+1}\right] ; X\right)} \leq r$ for every $i \geq 1$.

Next, for $i \geq 1$ and $t \in\left(t_{i}, s_{i}\right]$, we get

$$
\begin{aligned}
\|\Gamma u(t)\| \leq & \left\|u_{0}\right\|+\frac{1}{\alpha \Gamma(\alpha)} \sum_{i=0}^{N}\left(t_{i+1}-s_{i}\right)^{\alpha}\|f(s, u(s), \mathcal{B} u(s))\| \\
& +\frac{1}{\Gamma(\beta)} \sum_{i=0}^{N} \int_{t_{i}}^{s_{i}}\left(s_{i}-s\right)^{\beta-1}\left(\left\|g_{i}(s, u(s))-g_{i}(s, 0)\right\|+\left\|g_{i}(s, 0)\right\|\right) d s \\
& +\frac{1}{\Gamma(\beta)} \int_{t_{i}}^{t}(t-s)^{\beta-1}\left(\left\|g_{i}(s, u(s))-g_{i}(s, 0)\right\|+\left\|g_{i}(s, 0)\right\|\right) d s \\
& +\int_{t_{i}}^{t} \phi_{A}(t-s)\left(\left\|u_{0}\right\|+\frac{1}{\alpha \Gamma(\alpha)} \sum_{i=0}^{N}\left(t_{i+1}-s_{i}\right)^{\alpha}\|f(\tau, u(\tau), \mathcal{B} u(\tau))\|\right. \\
& +\frac{1}{\Gamma(\beta)} \sum_{i=0}^{N} \int_{t_{i}}^{s_{i}}\left(s_{i}-\tau\right)^{\beta-1}\left(\left\|g_{i}(\tau, u(\tau))-g_{i}(\tau, 0)\right\|+\left\|g_{i}(\tau, 0)\right\|\right) d \tau \\
& \left.+\frac{1}{\Gamma(\beta)} \int_{t_{i}}^{s}(s-\tau)^{\beta-1}\left(\left\|g_{i}(\tau, u(\tau))-g_{i}(\tau, 0)\right\|+\left\|g_{i}(\tau, 0)\right\|\right) d \tau\right) d s \\
\leq & \left(1+\left\|\phi_{A}\right\|_{L^{1}\left(\left(t_{i}, s_{i}\right] ; \mathbb{R}^{+}\right)}\right)\left(\left\|u_{0}\right\|+\frac{\sum_{i=0}^{N}\left(t_{i+1}-s_{i}\right)^{\alpha}}{\alpha \Gamma(\alpha)}\left\|m_{f}\right\| W(\|u\|+\|\mathcal{B} u\|)\right. \\
& \left.+\left(\frac{\sum_{i=0}^{N}\left(s_{i}-t_{i}\right)^{\beta}}{\beta \Gamma(\beta)}+\frac{\left(t-t_{i}\right)^{\beta}}{\beta \Gamma(\beta)}\right)\left(L_{g_{i}}\|u\|+\left\|g_{i}(\cdot, 0)\right\|\right)\right) \\
\leq & \left(1+\left\|\phi_{A}\right\|_{L^{1}\left(\left(t_{i}, s_{i}\right] ; \mathbb{R}^{+}\right)}\right)\left(\left\|u_{0}\right\|+\frac{\sum_{i=0}^{N}\left(t_{i+1}-s_{i}\right)^{\alpha}}{\alpha \Gamma(\alpha)}\left\|m_{f}\right\| W((1+K) r)\right. \\
& \left.+\left(\frac{\sum_{i=0}^{N}\left(s_{i}-t_{i}\right)^{\beta}}{\beta \Gamma(\beta)}+\frac{\left(s_{i}\right)^{\beta}}{\beta \Gamma(\beta)}\right)\left(L_{g_{i}} r+\left\|g_{i}(\cdot, 0)\right\|\right)\right) \\
\leq & r
\end{aligned}
$$

which implies that $\|\Gamma u\|_{C\left(\left(t_{i}, s_{i}\right] ; \mathbb{R}^{+}\right)} \leq r$.

In the similar way, we can proceed for $t \in\left[0, t_{1}\right]$,

$$
\begin{aligned}
\|\Gamma u\|_{C\left(\left(0, t_{1}\right] ; \mathbb{R}^{+}\right)} & \leq\left(\left\|u_{0}\right\|+\frac{t_{1}^{\alpha}}{\alpha \Gamma(\alpha)}\left\|m_{f}\right\| W((1+K) r)\right)\left(1+\left\|\phi_{A}\right\|_{L^{1}\left(\left(0, t_{1}\right] ; \mathbb{R}^{+}\right)}\right) \\
& \leq r
\end{aligned}
$$

From the above three inequalities, we infer that $\|\Gamma u(t)\|_{P C(X)} \leq r$ and $\Gamma$ has values in $B_{r}(0, P C(X))$.

To carry out the remaining proof, we introduce the decomposition map $\Gamma=\Gamma_{1}+\Gamma_{2}+\Gamma_{3}$, where $\Gamma_{i}: P C(X) \rightarrow P C(X), i=1,2,3$ are given by

$$
\left\|\Gamma_{1}\right\|=\left\{\begin{array}{l}
u_{0}+\int_{0}^{t} S^{\prime}(t-s) u_{0} d s, \quad t \in\left[0, t_{1}\right] \\
u_{0}+\frac{1}{\Gamma(\beta)} \sum_{i=0}^{N} \int_{t_{i}}^{s_{i}}\left(s_{i}-s\right)^{\beta-1} g_{i}(s, u(s)) d s \\
+\int_{s_{i}}^{t} S^{\prime}(t-s)\left(u_{0}+\frac{1}{\Gamma(\beta)} \sum_{i=0}^{N} \int_{t_{i}}^{s_{i}}\left(s_{i}-\tau\right)^{\beta-1} g_{i}(\tau, u(\tau)) d \tau\right) d s, t \in\left(s_{i}, t_{i+1}\right] \\
u_{0}+\frac{1}{\Gamma(\beta)} \sum_{i=0}^{N} \int_{t_{i}}^{s_{i}}\left(s_{i}-s\right)^{\beta-1} g_{i}(s, u(s)) d s+\frac{1}{\Gamma(\beta)} \int_{t_{i}}^{t}(t-s)^{\beta-1} g_{i}(s, u(s)) d s \\
+\int_{s_{i}}^{t} S^{\prime}(t-s)\left(u_{0}+\frac{1}{\Gamma(\beta)} \sum_{i=0}^{N} \int_{t_{i}}^{s_{i}}\left(s_{i}-\tau\right)^{\beta-1} g_{i}(\tau, u(\tau)) d \tau\right. \\
\left.+\frac{1}{\Gamma(\beta)} \int_{t_{i}}^{s}(s-\tau)^{\beta-1} g_{i}(\tau, u(\tau)) d \tau\right) d s, \quad t \in\left(t_{i}, s_{i}\right]
\end{array}\right.
$$




$$
\begin{aligned}
& \left\|\Gamma_{2}\right\|=\left\{\begin{array}{l}
\frac{1}{\Gamma(\alpha)} \int_{0}^{t}(t-s)^{\alpha-1} f(s, u(s), \mathcal{B} u(s)) d s, \quad t \in\left[0, t_{1}\right], \\
\frac{1}{\Gamma(\alpha)} \sum_{i=0}^{N} \int_{s_{i}}^{t_{i+1}}\left(t_{i+1}-s\right)^{\alpha-1} f(s, u(s), \mathcal{B} u(s)) d s \\
+\frac{1}{\Gamma(\alpha)} \int_{s_{i}}^{t}(t-s)^{\alpha-1} f(s, u(s), \mathcal{B} u(s)) d s, t \in\left(s_{i}, t_{i+1}\right] \\
\frac{1}{\Gamma(\alpha)} \sum_{i=0}^{N} \int_{s_{i}}^{t_{i+1}}\left(t_{i+1}-s\right)^{\alpha-1} f(s, u(s), \mathcal{B} u(s)) d s, \quad t \in\left(t_{i}, s_{i}\right],
\end{array}\right. \\
& \left\|\Gamma_{3}\right\|=\left\{\begin{array}{l}
\int_{0}^{t} S^{\prime}(t-s) \frac{1}{\Gamma(\alpha)} \int_{0}^{s}(s-\tau)^{\alpha-1} f(\tau, u(\tau), \mathcal{B} u(\tau)) d \tau d s, t \in\left[0, t_{1}\right], \\
\int_{s_{i}}^{t} S^{\prime}(t-s)\left(\frac{1}{\Gamma(\alpha)} \sum_{i=0}^{N} \int_{s_{i}}^{t_{i+1}}\left(t_{i+1}-\tau\right)^{\alpha-1} f(\tau, u(\tau), \mathcal{B} u(\tau)) d \tau\right. \\
\left.+\frac{1}{\Gamma(\alpha)} \int_{s_{i}}^{s}(s-\tau)^{\alpha-1} f(\tau, u(\tau), \mathcal{B} u(\tau)) d \tau\right), t \in\left(s_{i}, t_{i+1}\right] \\
\int_{t_{i}}^{t} S^{\prime}(t-s) \frac{1}{\Gamma(\alpha)} \sum_{i=0}^{N} \int_{s_{i}}^{t_{i+1}}\left(t_{i+1}-\tau\right)^{\alpha-1} f(\tau, u(\tau), \mathcal{B} u(\tau)) d \tau d s, t \in\left(t_{i}, s_{i}\right],
\end{array}\right.
\end{aligned}
$$

for all $i=1, \cdots, N$.

Now we divide the remainder of the proof into the following steps:

Step 1: The map $\Gamma_{1}$ is a contraction on $B_{r}(0, P C(X))$.

For $u, v \in B_{r}(0, P C(X)), i \in\{1, \cdots, N\}$ and $t \in\left(s_{i}, t_{i+1}\right]$, we get

$$
\begin{aligned}
\left\|\Gamma_{1} u(t)-\Gamma_{1} v(t)\right\| \leq & \frac{1}{\Gamma(\beta)} \sum_{i=0}^{N} \int_{t_{i}}^{s_{i}}\left(s_{i}-s\right)^{\beta-1}\left\|g_{i}(s, u(s))-g_{i}(s, v(s))\right\| d s \\
& +\int_{s_{i}}^{t} \phi_{A}(t-s)\left(\frac{1}{\Gamma(\beta)} \sum_{i=0}^{N} \int_{t_{i}}^{s_{i}}\left(s_{i}-\tau\right)^{\beta-1}\left\|g_{i}(\tau, u(\tau))-g_{i}(\tau, v(\tau))\right\| d \tau\right) \\
\leq & \frac{1}{\beta \Gamma(\beta)} \sum_{i=0}^{N}\left(s_{i}-t_{i}\right)^{\beta}\left\|L_{g_{i}}\right\|\left(1+\left\|\phi_{A}\right\|_{L^{1}\left(\left(s_{i}, t_{i+1}\right] ; \mathbb{R}^{+}\right)}\right)\|u-v\|_{P C(X)}
\end{aligned}
$$

Similarly for $t \in\left(t_{i}, s_{i}\right]$,

$$
\begin{aligned}
\left\|\Gamma_{1} u(t)-\Gamma_{1} v(t)\right\| \leq & \frac{1}{\Gamma(\beta)} \sum_{i=0}^{N} \int_{t_{i}}^{s_{i}}\left(s_{i}-s\right)^{\beta-1}\left\|g_{i}(s, u(s))-g_{i}(s, v(s))\right\| d s \\
& +\frac{1}{\Gamma(\beta)} \int_{t_{i}}^{t}(t-s)^{\beta-1}\left\|g_{i}(s, u(s))-g_{i}(s, v(s))\right\| d s \\
& +\int_{s_{i}}^{t} \phi_{A}(t-s)\left(\frac{1}{\Gamma(\beta)} \sum_{i=0}^{N} \int_{t_{i}}^{s_{i}}\left(s_{i}-\tau\right)^{\beta-1}\left\|g_{i}(\tau, u(\tau))-g_{i}(\tau, v(\tau))\right\| d \tau\right. \\
& \left.+\frac{1}{\Gamma(\beta)} \int_{t_{i}}^{s}(s-\tau)^{\beta-1}\left\|g_{i}(\tau, u(\tau))-g_{i}(\tau, v(\tau))\right\| d \tau\right) d s \\
\leq & \left(\frac{\sum_{i=0}^{N}\left(s_{i}-t_{i}\right)^{\beta}}{\beta \Gamma(\beta)}+\frac{s_{i}^{\beta}}{\beta \Gamma(\beta)}\right)\left\|L_{g_{i}}\right\|\left(1+\left\|\phi_{A}\right\|_{L^{1}\left(\left(t_{i}, s_{i}\right] ; \mathbb{R}^{+}\right)}\right)\|u-v\|_{P C(X)}
\end{aligned}
$$

which implies that $\left\|\Gamma_{1} u(t)-\Gamma_{1} v(t)\right\| \leq \Omega\|u-v\|_{P C(X)}$, where

$$
\begin{aligned}
\Omega= & \max \left\{\frac{1}{\beta \Gamma(\beta)} \sum_{i=0}^{N}\left(s_{i}-t_{i}\right)^{\beta}\left\|L_{g_{i}}\right\|\left(1+\left\|\phi_{A}\right\|_{L^{1}\left(\left(s_{i}, t_{i+1}\right] ; \mathbb{R}^{+}\right)}\right)\right), \\
& \left.\left(\frac{\sum_{i=0}^{N}\left(s_{i}-t_{i}\right)^{\beta}}{\beta \Gamma(\beta)}+\frac{s_{i}^{\beta}}{\beta \Gamma(\beta)}\right)\left\|L_{g_{i}}\right\|\left(1+\left\|\phi_{A}\right\|_{L^{1}\left(\left(t_{i}, s_{i}\right] ; \mathbb{R}^{+}\right)}\right)\right\}<1 .
\end{aligned}
$$

Hence, $\Gamma_{1}$ is a contraction on $B_{r}(0, P C([0,1] ; X))$.

Step 2: The map $\Gamma_{2}$ is completely continuous

From the properties of the function $f($.$) , it is easy to see that \Gamma_{2}$ is continuous.

Next, we show that $\Gamma_{2}$ is a compact operator on $B_{r}(0, P C([0,1] ; X))$. 
Consider, for $u \in B_{r}(0, P C(X))$ and $t \in\left(s_{i}, t_{i+1}\right]$,

$$
\begin{aligned}
\left\|\Gamma_{2} u(t+h)-\Gamma_{2} u(t)\right\| \leq & \frac{1}{\Gamma(\alpha)} \int_{s_{i}}^{t}\left((t-s)^{\alpha-1}-(t+h-s)^{\alpha-1}\right)\|f(s, u(s), \mathcal{B} u(s))\| d s \\
& +\frac{1}{\Gamma(\alpha)} \int_{t}^{t+h}(t+h-s)^{\alpha-1}\|f(s, u(s), \mathcal{B} u(s))\| d s \\
\leq & \frac{1}{\alpha \Gamma(\alpha)}\left(\left(t-s_{i}\right)^{\alpha}-\left(t+h-s_{i}\right)^{\alpha}\right)\|f(s, u(s), \mathcal{B} u(s))\| \\
& +\frac{1}{\alpha \Gamma(\alpha)} h^{\alpha}\|f(s, u(s), \mathcal{B} u(s))\| \\
\leq & \frac{2}{\alpha \Gamma(\alpha)} h^{\alpha}\|f(s, u(s), \mathcal{B} u(s))\|,
\end{aligned}
$$

In the same way we can get for $t \in\left(t_{i}, s_{i}\right]$,

$$
\left\|\Gamma_{2} u(t+h)-\Gamma_{2} u(t)\right\|=0
$$

and for $t \in\left[0, t_{1}\right]$,

$$
\left\|\Gamma_{2} u(t+h)-\Gamma_{2} u(t)\right\| \leq \frac{2}{\alpha \Gamma(\alpha)} h^{\alpha}\|f(s, u(s), \mathcal{B} u(s))\|
$$

which implies that $\left.\Gamma_{2} B_{r} \widetilde{(0, P C}(X)\right)$ is equicontinuous subset of $C\left(\left[t_{i}, t_{i+1}\right] ; X\right)$.

Next, we show that the set $\left\{\Gamma_{2} u(s): u \in B_{r}(0, P C([0,1] ; X)), s \in[0,1]\right\}$ is relatively compact in $X$.

Let $s_{i}<\epsilon<t \leq t_{i+1}$. From the mean value theorem for the Bochner integral (see [21, Lemma 2.1.3]), for $u \in B_{r}(0, P C(X))$, we see that

$$
\begin{aligned}
\Gamma_{2} u(t)= & \frac{1}{\Gamma(\alpha)} \int_{s_{i}}^{t-\epsilon} \frac{f(s, u(s), \mathcal{B} u(s))}{(t-s)^{\alpha-1}} d s+\frac{1}{\Gamma(\alpha)} \int_{t-\epsilon}^{t} \frac{f(s, u(s), \mathcal{B} u(s))}{(t-s)^{\alpha-1}} d s \\
& +\frac{1}{\Gamma(\alpha)} \sum_{i=0}^{N} \int_{s_{i}}^{t_{i+1}} \frac{f(s, u(s), \mathcal{B} u(s))}{(t-s)^{\alpha-1}} d s \\
\in & B_{\frac{r_{1} \epsilon^{\alpha}}{\alpha \Gamma(\alpha)}}(0, X)+B_{d 1}(0, X) \\
& +\frac{\left(t-\epsilon-s_{i}\right)}{\Gamma(\alpha)} \frac{\operatorname{co}\left(\left\{(t-s)^{\alpha-1} f(s, u(s), \mathcal{B} u(s)): s \in\left[s_{i}, t-\epsilon\right]\right\}\right)}{}
\end{aligned}
$$

where $d 1=\frac{r_{1} \sum_{i=0}^{N}\left(t_{i+1}-s_{i}\right)^{\alpha}}{\alpha \Gamma(\alpha)}$. Since the map $i_{c}$ is compact and $f \in C([0,1] ; \mathcal{D})$, from the above inclusion we obtain that

$$
\left\{\Gamma_{2} u(t): u \in B_{r}(0, P C(X)), s \in\left[\epsilon, t_{i+1}\right]\right\} \subset B_{\frac{r_{1} \epsilon^{\alpha}}{\alpha \Gamma(\alpha)}}(0, X)+B_{d 1}(0, X)+K_{\epsilon},
$$

where $r_{1}=\left\|m_{f}\right\|_{C\left(\left(s_{i}, t_{i+1}\right] ; \mathbb{R}^{+}\right)} W((1+K) r)$ and $K_{\epsilon}$ is a compact subset of $X$. Moreover, by using

$$
\left\{\Gamma_{2} u(t): u \in B_{r}(0, P C(X)), s \in\left[s_{i}, \epsilon\right]\right\} \subset B_{\frac{r_{1} \epsilon^{\alpha}}{\alpha \Gamma(\alpha)}}(0, X)+B_{d 1}(0, X),
$$

we find that

$$
\begin{aligned}
& \left\{\Gamma_{2} u(t): u \in B_{r}(0, P C(X)), s \in\left(s_{i}, t_{i+1}\right]\right\} \\
& \quad \subset B_{\frac{r_{1} \epsilon^{\alpha}}{\alpha \Gamma(\alpha)}}(0, X)+B_{d 1}(0, X) \cup B_{\frac{r_{1} \epsilon^{\alpha}}{\alpha \Gamma(\alpha)}}(0, X)+B_{d 1}(0, X)+K_{\epsilon}
\end{aligned}
$$

which gives relatively compact in $X$. Since $B_{d 1}(0, X)$ is relatively compact and $\operatorname{Diam}\left(B_{\frac{r_{1} \epsilon^{\alpha}}{\alpha \Gamma(\alpha)}}(0, X)\right) \rightarrow 0$ as $\epsilon \rightarrow 0$. 
From the above procedure we can show that $\Gamma_{2}$ is relatively compact for the remaining intervals $\left(0, t_{1}\right]$ and $\left(t_{i}, s_{i}\right]$.

These results can be used to conclude that $\Gamma_{2}$ is completely continuous.

Step 3: The set $\left\{\Gamma_{3} u(t): u \in B_{r}(0, P C([0,1] ; X))\right\}$ is relatively compact in $X$ for all $t \in[0,1]$.

Let $s_{i}<\epsilon<t \leq t_{i+1}$ and $r_{2}=\left\|m_{f}\right\|_{C\left(\left[s_{i}, t_{i+1}\right] ; \mathbb{R}^{+}\right)} W((1+K) r)\left\|\phi_{A}\right\|_{L^{1}\left((t-\epsilon, t] ; \mathbb{R}^{+}\right)}$. The set $U=\left\{\Gamma_{2} u(t)\right.$ : $\left.u \in B_{r}(0, P C(X))\right\}$ is relatively compact in $X$, which is according to Step 2 . If $u \in B_{r}(0, P C([0,1] ; X))$, from the mean value theorem for the Bochner integral we get

$$
\begin{aligned}
\Gamma_{3} u(t) & =\int_{s_{i}}^{t-\epsilon} S^{\prime}(t-s) \Gamma_{2} u(s) d s+\int_{t-\epsilon}^{t} S^{\prime}(t-s) \Gamma_{2} u(s) d s \\
& \subset\left(t-\epsilon-s_{i}\right) \overline{\operatorname{co}\left(\left\{S^{\prime}(s) x: s \in[\epsilon, t], x \in \bar{U}\right\}\right)}+B_{d 2}(0, X)
\end{aligned}
$$

where $d 2=\frac{r_{2}\left(\epsilon^{\alpha}+\sum_{i=0}^{N}\left(t_{i+1}-s_{i}\right)^{\alpha}\right)}{\alpha \Gamma(\alpha)}$ and hence, $\left\{\Gamma_{3} u(t): u \in B_{r}(0, P C([0,1] ; X))\right\} \subset K_{\epsilon}+B_{d 2}(0, X)$ is relatively compact in $X$, since $K_{\epsilon}$ is compact and $\operatorname{Diam}\left(B_{d 2}(0, X)\right) \rightarrow 0$ as $\epsilon \rightarrow 0$.

Also $\Gamma_{3}$ is relatively compact in $X$ for $\left(0, t_{1}\right]$ and for $\left(t_{i}, s_{i}\right], i=1, \cdots, N$.

In the sequel we need to prove that the set of functions $\left.\Gamma_{3} B_{r} \widetilde{(0, P C}(X)\right)$ is an equicontinuous subset of $C\left(\left[t_{i}, t_{i+1}\right] ; X\right)$.

Following from Step 2 , it is obvious that $\left.\Gamma_{3} B_{r} \widetilde{(0, P C}(X)\right)$ is right equicontinuous on $\left(t_{i}, s_{i}\right)$ and left equicontinous on $\left(t_{i}, s_{i}\right]$.

Assume that $t \in\left(s_{i}, t_{i+1}\right), i=0,1, \cdots, N$ and $S^{\prime}$ is continuous such that $\left\|S^{\prime}(t)-S^{\prime}(s)\right\|<\epsilon$. For $u \in B_{r}(0, P C([0,1] ; X))$ and let $0<h<\epsilon$ such that $t<t+h$, we get

$$
\begin{aligned}
& \left\|\Gamma_{3} u(t+h)-\Gamma_{3} u(t)\right\| \\
& \quad \leq \int_{s_{i}}^{t}\left\|S^{\prime}(t+h-s)-S^{\prime}(t-s)\right\|\left(\frac{1}{\Gamma(\alpha)} \sum_{i=0}^{N} \int_{s_{i}}^{t_{i+1}}\left(t_{i+1}-\tau\right)^{\alpha-1}\|f(\tau, u(\tau), \mathcal{B} u(t))\| d \tau\right. \\
& \left.\quad+\frac{1}{\Gamma(\alpha)} \int_{s_{i}}^{s}(s-\tau)^{\alpha-1}\|f(\tau, u(\tau), \mathcal{B} u(t))\| d \tau\right) d s \\
& \quad+\int_{t}^{t+h}\left\|S^{\prime}(t+h-s)\right\|\left(\frac{1}{\Gamma(\alpha)} \sum_{i=0}^{N} \int_{s_{i}}^{t_{i+1}}\left(t_{i+1}-\tau\right)^{\alpha-1}\|f(\tau, u(\tau), \mathcal{B} u(t))\| d \tau\right. \\
& \left.\quad+\frac{1}{\Gamma(\alpha)} \int_{s_{i}}^{s}(s-\tau)^{\alpha-1}\|f(\tau, u(\tau), \mathcal{B} u(t))\| d \tau\right) d s \\
& \quad \leq \frac{\epsilon t_{i+1}}{\alpha \Gamma(\alpha)}\left(\sum_{i=0}^{N}\left(t_{i+1}-s_{i}\right)^{\alpha}+t_{i+1}^{\alpha}\right)\left\|m_{f}\right\| W((1+K) r) \\
& \quad+\frac{1}{\alpha \Gamma(\alpha)}\left(\sum_{i=0}^{N}\left(t_{i+1}-s_{i}\right)^{\alpha}+t_{i+1}^{\alpha}\right)\left\|m_{f}\right\| W((1+K) r)\left\|\phi_{A}\right\|_{L^{1}\left([0, h] ; \mathbb{R}^{+}\right)}
\end{aligned}
$$

which implies that $\left.\Gamma_{3} B_{r} \widetilde{(0, P C}(X)\right)$ is right equicontinuous at $t \in\left(s_{i}, t_{i+1}\right)$. In a similar way we can prove that $\left.\Gamma_{3} B_{r} \widehat{(0, P C}(X)\right)$ is left equicontinuous at $t \in\left(s_{i}, t_{i+1}\right]$ and right equicontinuous at $s_{i}$. Thus, $\left.\Gamma_{3} B_{r} \widetilde{(0, P C}(X)\right)$ is equicontinuous on $\left(s_{i}, t_{i+1}\right]$.

Next, from the above argument we can easily say that $\left.\Gamma_{3} B_{r} \widetilde{(0, P C}(X)\right)$ is equicontinuous on $\left(0, t_{1}\right]$. Hence from all the above all intervals we conclude that $\left.\Gamma_{3} B_{r} \widetilde{(0, P C}(X)\right)$ is a equicontinuous subset of $C\left(\left[t_{i}, t_{i+1}\right] ; X\right)$.

From the above steps and Lemma 21 it follows that $\Gamma_{1}$ is contraction, $\Gamma_{2}$ and $\Gamma_{3}$ are completely continuous. Thus, $\Gamma$ is a condensing operator from $B_{r}(0, P C(X))$ into $B_{r}(0, P C(X))$ and from [21, Theorem 4.3.2] we infer that there exists a mild solution for the problem (1)-(3). 


\section{Application}

To study the existence of solutions for the partial differential equations with fractional temporal derivative, we may take the space $X=L^{2}([0, \pi])$ and let $A$ be the operator given by $A x=x^{\prime \prime}$ with domain $\mathcal{D}(A)=\left\{x \in X: x^{\prime \prime} \in X, x(0)=x(\pi)=0\right\}$. It is well-known that $A$ is the infinitesimal generator of an analytic semigroup $(T(t))_{t \geq 0}$ on $X$.

Now, consider the following partial fractional impulsive and nonlocal equations:

$$
\begin{aligned}
{ }^{c} D^{\alpha} u(t, \zeta)= & \frac{\partial^{2}}{\partial \zeta^{2}} u(t, \zeta)+a_{1}(t)+a_{2}(u(t, \zeta)) \\
& +\int_{0}^{t} a_{3}(t-s) u(s, \zeta),(t, \zeta) \in \cup_{i=1}^{N}\left(s_{i}, t_{i+1}\right] \times[0, \pi] \\
u(t, 0)= & u(t, \pi)=0, \quad t \in[0, a] \\
u(0, \zeta)= & z(\zeta) \zeta \in[0, \pi] \\
{ }^{c} D^{\alpha} u(t, \zeta)= & H_{i}(t, u(t, \zeta)), \zeta \in[0, \pi], t \in\left(t_{i}, s_{i}\right], i=1, \cdots, N
\end{aligned}
$$

where $0=t_{0}=s_{0}<t_{1}<s_{1}<\cdots<t_{N}<s_{N}<t_{N+1}=1$ are fixed real numbers, $\alpha \in(0,1), z \in X, a_{j} \in C\left([0,1] \times \mathbb{R}^{+} ; \mathbb{R}^{+}\right), j=1,2,3, H_{i} \in C\left(\left(t_{i}, s_{i}\right] \times \mathbb{R}^{+} ; \mathbb{R}^{+}\right)$for all $i=1, \cdots, N$.

The operator $A$ has discrete spectrum with eigenvalues of the form $-n^{2}, n \in \mathbb{N}$, and corresponding normalized eigenfunctions given by $z_{n}(\zeta)=\left(\frac{2}{\pi}\right)^{1 / 2} \sin (n \zeta)$. In addition, $\left\{z_{n}: n \in \mathbb{N}\right\}$ is an orthonormal basis for $X, T(t) x=\sum_{n=1}^{\infty} \exp ^{-n^{2} t}<x, z_{n}>z_{n}$ for all $x \in X$ and for every $t>0$. From these expressions it follows that $(T(t))_{t \geq 0}$ is a uniformly bounded compact semigroup, so that, $R(\lambda, A)=(\lambda-A)^{-1}$ is a compact operator for all $\lambda \in \rho(A)$.

To represent the fractional impulsive equations (9)-(12) in the abstract form (1)-(3), we consider the functions $B(t, s): X \rightarrow X, \mathcal{B}: C([0,1] ; X) \rightarrow X, f:[0,1] \times X^{2} \rightarrow X$, and $h_{i}:\left(t_{i}, s_{i}\right] \times X \rightarrow X$ defined by $B(t, s) x=a_{3}(t-s) x, \mathcal{B} u(t)=\int_{0}^{t} B(t, s) u(s) d s$ and

$$
\begin{aligned}
f(t, u, \mathcal{B} u)(\zeta) & =a_{1}(\zeta)+a_{2}(u(t, \zeta))+\mathcal{B} u(t), \\
g_{i}(t, u)(\zeta) & =H_{i}(t, u(t, \zeta))
\end{aligned}
$$

and $B(t, s)$ and $\mathcal{B}($.$) are bounded linear operators.$

Next, from [28, Chap. 2] we know that the integral equation,

$$
u(t)=\frac{1}{\Gamma(\alpha)} \int_{0}^{t}(t-s)^{\alpha-1} A u(s) d s, t \in\left[0, t_{1}\right]
$$

has an associated analytic resolvent operator $(S(t))_{t \geq 0}$ on $X$ which is given by

$$
S(t)=\left\{\begin{array}{l}
\frac{1}{2 \pi i} \int_{\Gamma_{r, \theta}} e^{\lambda t}\left(\lambda^{\alpha}-A\right)^{-1} \lambda^{\alpha-1} d \lambda, t \in\left(0, t_{1}\right] \\
I, \quad t=0
\end{array}\right.
$$

Similarly, for the integral equation,

$$
u(t)=\sum_{i=0}^{N} \frac{1}{\Gamma(\alpha)} \int_{s_{i}}^{t_{i+1}}\left(t_{i+1}-s\right)^{\alpha-1} A u(s) d s+\frac{1}{\Gamma(\alpha)} \int_{0}^{t}(t-s)^{\alpha-1} A u(s) d s, t \in\left(s_{i}, t_{i+1}\right],
$$

has an associated analytic resolvent operator $(S(t))_{t \geq 0}$ on $X$ which is given by

$$
S(t)=\frac{1}{2 \pi i} \int_{\Gamma_{r, \theta}} e^{\lambda t}\left(\lambda^{\alpha}-A\right)^{-1} \lambda^{\alpha-1}\left(1+A \sum_{i=0}^{N} \int_{s_{i}}^{t_{i+1}}\left(t_{i+1}-s\right)^{\alpha-1} S(s) d s\right) d \lambda
$$

Finally, we can easily get the resolvent operator for the integral equation

$$
u(t)=\sum_{i=0}^{N} \frac{1}{\Gamma(\alpha)} \int_{s_{i}}^{t_{i+1}}\left(t_{i+1}-s\right)^{\alpha-1} A u(s) d s,
$$


where $\Gamma_{r, \theta}$ denotes the contour consisting of the rays $\left\{r e^{i \theta}: r \geq 0\right\}$ and $\left\{r e^{-i \theta}: r \geq 0\right\}$ for some $\theta \in(\pi, \pi / 2)$. The given resolvent operator $S(t)$ is compact for all $t>0$, since $(\lambda-A)^{-1}$ is compact.

Now, $u \in P C(X)$ is a mild solution of (9)-(12), if $u($.$) is a mild solution of the associated abstract$ problem (1)-(3). Suppose that the functions $f, g_{i}$ satisfy the hypotheses (H1)-(H2), there exists a mild solution $u \in P C(X)$ of (9)-(12) in view of Theorem 34.

\section{References}

1. A. Anguraj, M. Mallika Arjunan, "Existence and uniqueness of mild and classical solutions of impulsive evolution equations," Elect. J. Diff. Eqns., vol. 111, pp. 1-8, 2005.

2. A. Anguraj, K. Karthikeyan, "Existence of solutions for impulsive neutral functional differential equations with nonlocal conditions," Nonlinear Anal., vol. 70, pp. 2717-2721, 2009.

3. Y.K. Chang, A. Anguraj and M. Mallika Arjunan, "Existence results for impulsive neutral functional differential equations with delay," Nonlinear Analysis: Hybrid system, vol. 2no. 1, pp. 209-218, 2008.

4. A. Anguraj, M. Lathamaheshwari, "Existence results for fractional differential equations with infinite delay and interval impulsive conditions," Malaya J. Mat., vol. 2 no. 1, pp. 16-23, 2014.

5. M. Belmekki, M. Benchohra, "Existence results for fractional order semilinear functional differential equations with nondense domain," Nonlinear Anal., vol. 72, pp. 925-932, 2010.

6. A. Chandha, D.N. Pandey, "Existence of the mild solution for impulsive semilinear differential equation," Int. J. of Part. Diff. Eqns., Article ID 640931, 8 pages, 2014.

7. D.Delbosco, L. Rodino, "Existence and uniqueness for a nonlinear fractional differential equations," $J$. of Math. Anal. Appl., vol. 204, pp. 609-625, 1996.

8. D. Delbosco, "Fractional calculus and function spaces," J. Fract. Cal., vol. 6, pp. 45-53, 1994.

9. Z. Fan, Gang Li, "Existence results for semilinear differential equations with nonlocal conditions," $J$. Funct. Anal., vol. 258, pp. 1709 - 1727, 2010.

10. Ganga Ram Gautam, Jaydev Dabas, "Existence result of fractional functional integro-differential equation with not instantaneous impulse," Int. J. Adv. Appl. Math. and Mech., vol. 1 no. 3, pp. 11 - 21, 2014.

11. E. Hernández, M. Pierri, G. Goncalves, "Existence results for an impulsive abstract partial differential equations with state-dependent delay," Comput. Math. Appl., vol. 52, pp. 411 - 420, 2006.

12. E. Hernández, D. O'Regan, "On a new class of abstract impulsive differential equations," Proceedings of the American Mathematical Society, vol. 141, pp. 1641 - 1649, 2013.

13. E. Hernández, D. O'Regan, K. Balachandran, "On recent developments in the theory of abstract differential equations with fractional derivatives," Nonlinear Analysis, vol. 73, pp. 3462-3471, 2010.

14. E. Hernández, D. O'Regan, K. Balachandran, "Existence results for abstract fractional differential equations with nonlocal conditions via resolvent operators," Indagationes mathematicae, vol. 24, pp. 68 - 82, 2013.

15. O.K. Jaradat, A. Al-Omari, S. Momani, "Existence of mild solutions for fractional semilinear initial value problems," Nonlinear Anal., vol. 69 no. 9, pp. 3153 - 3159, 2008.

16. A.A. Kilbas, H.M. Srivastava, J.J. Trujillo, "Theory and application of fractional differential equations," North-Holland, Amsterdam, 2006.

17. N. Kosmatov, "Initial value problems of fractional order with fractional impulsive conditions, "Results in Math., vol. 63, pp. 1289 - 1310, 2013.

18. V. Lakshmikantham, "Theory of fractional functional differential equations," Nonlinear Analysis, vol. 69, pp. 3337-3343, 2008.

19. V. Lakshmikantham, DD. Bainov, P.S. Simeonov, "Theory of impulsive differential equations," World Scientific, Singapore, 1989.

20. James H. Liu, "Nonlinear impulsive evolution equations," Dynam. Contin. Discrete Impuls. Systems vol. 6, pp. 77-85, 1999.

21. R.H. Martin, "Nonlinear operators and differential equations in Banach spaces," Robert E. Krieger publ. co., Florida, 1987.

22. K.S. Miller and B. Ross, "An introduction to the fractional calculus and fractional differential equations," Wiley, New York, 1993.

23. G.M. Mophou, "Existence and uniqueness of mild solutions to impulsive fractional differential equations," Nonlinear Anal., vol. 72, pp. 1604-1615, 2010.

24. J.J. Nieto, D. O'Regan, "Variational approach to impulsive differential equations," Nonlinear Anal., Real World Appl., vol. 10, pp. 680-690, 2009. 
25. D.N. Pandey, A. Ujlayan, D. Bahuguna, "On a solution to fractional order integrodifferential equations with analytic semigroups," Nonlinear Anal., vol. 71, pp. 3690-3698, 2009.

26. M. Pierri, Donal O'Regan, V. Rolink, "Existence of solutions for semi-linear absract differential equations with not instantaneous impulses," Applied mathematics and computation, vol. 219, pp. 6743 - 6749, 2013.

27. I. Podlubny, "Fractional differential equations," Mathematics in Sciences and Engineering, Academic press, San Diego, 1999.

28. J. Prüss, "Evolutionary integral equations and Applications," Monographs in Mathematics, Springer, 2012.

29. JinRong Wang, Xuezhu Li, "Periodic BVP for integer/fractional order nonlinear differential equations with non-instantaneous impulses," J. Appl. Math. Comput., vol. 46, pp. 321 - 334, 2014.

30. JinRang Wang, Y. Zhou, Z. Lin, "On a new class of impulsive fractional differential equations," Appl. Math. and Comput., vol. 242, pp. 649 - 657, 2014. 